\title{
Experience-dependent regulation of presynaptic NMDARs enhances neurotransmitter release at neocortical synapses
}

\author{
Joanna Urban-Ciecko, ${ }^{1,3}$ jing A. Wen, ${ }^{1,3}$ Puja K. Parekh, ${ }^{2}$ and Alison L. Barth ${ }^{1}$ \\ ${ }^{1}$ Department of Biological Sciences and Center for the Neural Basis of Cognition, Carnegie Mellon University, Pittsburgh, Pennsylvania \\ 15213, USA; ${ }^{2}$ Center for Neuroscience at the University of Pittsburgh, Department of Psychiatry, Pittsburgh, Pennsylvania 15219, USA
}

\begin{abstract}
Sensory experience can selectively alter excitatory synaptic strength at neocortical synapses. The rapid increase in synaptic strength induced by selective whisker stimulation (single-row experience/SRE, where all but one row of whiskers has been removed from the mouse face) is due, at least in part, to the trafficking of AMPA receptors (AMPARs) to the post-synaptic membrane, and is developmentally regulated. How enhanced sensory experience can alter presynaptic release properties in the developing neocortex has not been investigated. Using paired-pulse stimulation at layer 4-2/3 synapses in acute brain slices, we found that presynaptic release probability progressively increases in the spared-whisker barrel column over the first $24 \mathrm{~h}$ of SRE. Enhanced release probability can be at least partly attributed to presynaptic NMDA receptors (NMDARs). We find that the influence of presynaptic NMDARs in enhancing EPSC amplitude markedly increases during SRE. This occurs at the same time when recently potentiated synapses become highly susceptible to a NMDAR-dependent form of synaptic depression, during the labile phase of plasticity. Thus, these data show that augmented sensory stimulation can enhance release probability at layer 4-2/3 synapses and enhance the function of presynaptic NMDARs. Because presynaptic NMDARs have been linked to synaptic depression at layer 4-2/3 synapses, we propose that SRE-dependent up-regulation of presynaptic NMDARs is responsible for enhanced synaptic depression during the labile stage of plasticity.
\end{abstract}

[Supplemental material is available for this article.]

Sensory experience can enhance the spike output of neocortical neurons, a phenomenon that has been widely investigated at a cellular and molecular level across multiple brain areas. Experience-dependent potentiation of neuronal responses has been well characterized in somatosensory cortex (S1), where synaptic potentiation, the addition of new synaptic contacts, and a reduction in local inhibition all play a role in transforming the output of neural circuits (Finnerty et al. 1999; Knott et al. 2002; Barth et al. 2004; Clem and Barth 2006; Jiao et al. 2006; Cheetham et al. 2007; Wen and Barth 2011; Jacob et al. 2012). During postnatal development, experience-dependent plasticity is enhanced, and there has been great interest in understanding the cellular and molecular mechanisms that regulate this phenomenon in order to facilitate learning in other training paradigms as well as to restore plasticity in adults (Feldman et al. 1998; Pizzorusso et al. 2002; Hensch 2005; Di Cristo et al. 2007; Morishita et al. 2010; Gu et al. 2013; Kuhlman et al. 2013).

At layer 4-2/3 synapses of primary somatosensory (barrel) cortex in mice, experience-dependent glutamatergic synaptic strengthening is associated with the trafficking of post-synaptic AMPARs. In contrast to activity-dependent changes in postsynaptic measures of synaptic strength in primary sensory cortex, presynaptic changes have been less well characterized. Although sensory deprivation can decrease release probability at glutamatergic synapses in neocortical areas representing the deprived inputs (Bender et al. 2006a), it is unknown whether selective sen-

\footnotetext{
${ }^{3}$ These authors contributed equally to this work.

Corresponding author: barth@cmu.edu

Article is online at http://www.learnmem.org/cgi/doi/10.1101//m.035741.114. Freely available online through the Learning \& Memory Open Access option.
}

sory stimulation can enhance release. Indeed, it is conceivable that post-synaptic potentiation is accompanied by a reduction in release probability, as a homeostatic adjustment to the increased activity of this pathway (Turrigiano et al. 1998).

Here we sought to investigate whether whisker-driven activity in barrel cortex could alter presynaptic release properties at layer 4-2/3 synapses. We focused on SRE-driven plasticity at the end of the second post-natal week, since the timecourse and receptor subtypes required for post-synaptic changes in this experimental paradigm are well characterized. During SRE, layer 4-2/3 synapses in the whisker-spared barrel columns show a rapid NMDAR-dependent increase in strength over the first $12 \mathrm{~h}$, nearly doubling in amplitude (Wen et al. 2013). Potentiated layer 4-2/3 synapses then undergo an input-dependent reduction in synaptic strength also linked to NMDAR activation during the labile phase, that lasts $\sim 12 \mathrm{~h}$ before changes become stabilized (Wen et al. 2013).

The transition from NMDAR-dependent potentiation to NMDAR-dependent depression during SRE is a form of synaptic metaplasticity that can be observed both in vivo and in vitro (Clem et al. 2008). The mechanistic basis for this SRE-dependent change in NMDAR function remains obscure. Because NMDARs can occur both pre- and post-synaptically at layer 4-2/3 synapses, modification at both sites is possible (Corlew et al. 2008).

Presynaptic NMDARs have been anatomically observed at neocortical synapses, where they are localized to axonal boutons

(C) 2014 Urban-Ciecko et al. This article, published in Learning \& Memory, is available under a Creative Commons License (Attribution-NonCommercial 4.0 International), as described at http://creativecommons.org/licenses/ by-nc/4.0/. 
by immunoelectron microscopy (EM; Aoki et al. 1994; Charton et al. 1999; Corlew et al. 2007; Larsen et al. 2011). Putatively presynaptic NMDARs (referred to as presynaptic NMDARs for clarity) regulate the frequency of miniature EPSCs in layer 2/3 neurons in early post-natal neocortex (Berretta and Jones 1996; Brasier and Feldman 2008; Kunz et al. 2013), suggesting that they are gated by ambient glutamate even in the absence of bursting activity. Intracellular administration of NMDAR-antagonists into presynaptic layer 4 neurons is sufficient to block spike-timing-dependent long-term depression (t-LTD) at layer 4-2/3 synapses (RodríguezMoreno and Paulsen 2008), and t-LTD is developmentally regulated (Banerjee et al. 2009; Itami and Kimura 2012). Because SRE transiently shifts NMDAR-activation to favor synaptic depression (Clem et al. 2008; Wen and Barth 2012; Wen et al. 2013), and because t-LTD at layer $4-2 / 3$ synapses requires presynaptic NMDARS, we hypothesized that these receptors might be functionally up-regulated during the labile phase of SRE-induced plasticity.

Initially, we examined how SRE can alter the paired-pulse ratio (PPR) during the initiation, labile, and stabilization phases of experience-dependent plasticity in S1. PPR slowly decreases over the initial $24 \mathrm{~h}$ of SRE, indicating a progressive enhancement of synaptic release properties at layer $4-2 / 3$ synapses. We next investigated whether SRE could alter the influence of presynaptic NMDARs. In control animals under our experimental conditions, presynaptic NMDARs minimally influence the amplitude of isolated, evoked layer 4-2/3 EPSCs. However, SRE induces a significant increase in the influence of presynaptic NMDARs on EPSC amplitude during both the initiation and labile phases without simultaneously altering the PPR. These data show that presynaptic NMDARs can be regulated by sensory input and that they can influence EPSC amplitude by two different pathways.

\section{Results}

\section{Presynaptic release probability is enhanced by whisker activity}

Mice aged P12-13 were subjected to removal of all but the D-row of whiskers for variable time intervals, and acute brain slices were prepared that allowed identification of the spared D-row (Fig. 1A,B). Layer 4-2/3 EPSCs were evoked using extracellular stimulation in the center of a layer 4 barrel (Fig. 1C), and paired stimuli were delivered at $30 \mathrm{~Hz}$ (Fig. 1D). Previous studies have shown that this SRE manipulation is sufficient to increase the quantal amplitude of layer 4-2/3 EPSCs (Clem and Barth 2006; Clem et al. 2010; Wen et al. 2013). In the control, whisker-intact animals, mean PPR is on average modestly depressing (Fig. 1E; $0.92+0.06 ; n=$ 22 cells and 14 mice), consistent with prior studies in young animals (Reyes et al. 1998; Rozov et al. 2001; Bender et al. 2006a,b; Brasier and Feldman 2008). The range of PPR values for individual cells from control animals varied considerably, where some synapses were strongly facilitating and others profoundly depressing (PPR range $=1.44$, indicative of a strongly facilitating synapse, versus 0.15 , a strongly depressing synapse, $n=22$ ).

Over the course of $24 \mathrm{~h}$ of SRE, we observed a slow decrease in mean PPR at layer 4-2/3 synapses, whereby $24 \mathrm{~h}$ after the SRE onset it had dropped to $0.66+0.03$ (Fig. $1 \mathrm{E} ; n=17$ cells in 12 mice; $P=0.02$ by Kruskal-Wallis with Dunn's multiple comparison). This is typically interpreted as an increase in presynaptic release probability (Fig. 1E). These effects were independent of the recording temperature used (Supplemental Fig. 1). In addition, SRE led to a decrease in the overall variance of the PPR, where PPR values for individual cells became more similar by $24 \mathrm{~h}$ (PPR range $0.88-$ $0.39, n=17$ cells in 12 mice). This phenomenon suggests that changes across individual cells might be asynchronous, but converge to a common state where release probability is enhanced.
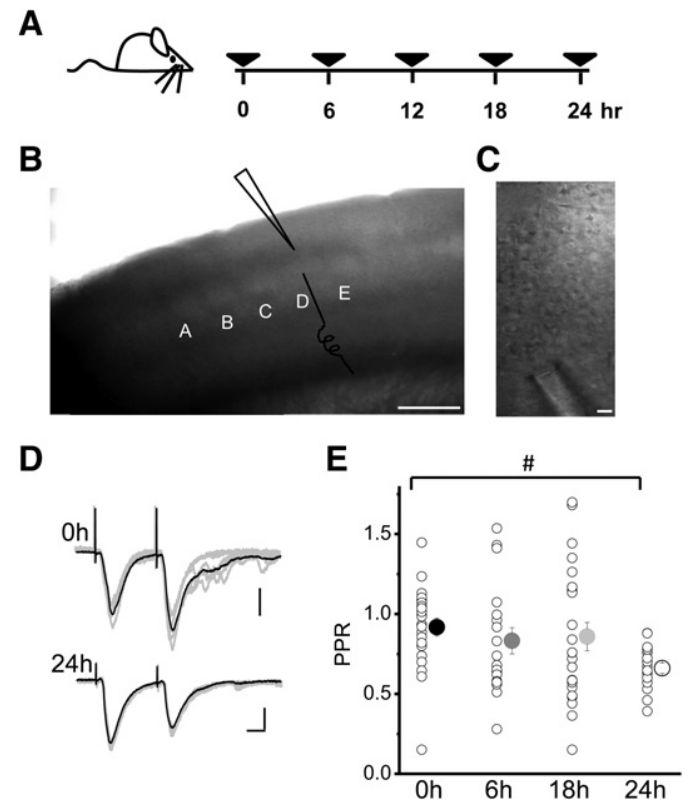

Figure 1. Presynaptic release probability is enhanced by whisker activity. $(A)$ Schematic of time points recording after whisker plucking. (B) Bright-field image of recording configuration in $D$ barrel column with the schematic of stimulating and recording electrode placement for measuring layer 4-2/3 EPSCs. Scale bar, $500 \mu \mathrm{m}$. (C) The same as $B$ but higher magnification. Scale bar, $20 \mu \mathrm{m}$. (D) Average traces of evoked EPSCs recorded at layer 4-2/3 synapses for control and animals after $24 \mathrm{~h}$ of SRE. Gray traces are individual responses. Calibration: $50 \mathrm{pA}, 10 \mathrm{msec}$. (E) Scatter plot of PPR from individual cells recorded at $0 \mathrm{~h}(n=22$ cells and 14 animals), $6 \mathrm{~h}(n=18$ cells and 14 animals), $18 \mathrm{~h}(n=23$ cells and 16 animals) and $24 \mathrm{~h}$ ( $n=17$ cells and 12 animals) of SRE. ( $\left.{ }^{\#}\right) P>$ 0.05 LMM, $P<0.05$ ANOVA.

\section{Presynaptic NMDARs at layer 4-2/3 synapses}

There is strong evidence for presynaptic NMDARs at layer 4-2/3 synapses in the developing somatosensory cortex, where these receptors have been shown to modulate basal EPSC amplitude, decrease the PPR (Sjöstrom et al. 2003; Bender et al. 2006a; Brasier and Feldman 2008; Rodríguez-Moreno et al. 2013). There is also anatomical evidence using immuno-EM that indicates NMDARs can be found at presynaptic terminals in the neocortex (Aoki et al. 1994; Larsen et al. 2011). Typically, detection of a presynaptic NMDAR effect on release properties has also required specific experimental manipulations to enhance their function: for example, blockade of glutamate reuptake using TBOA or presynaptic bursting activity (Bender et al. 2006b; Brasier and Feldman 2008; Rodríguez-Moreno et al. 2013). In the absence of these manipulations, the influence of presynaptic NMDARs in regulating the post-synaptic EPSC has been hard to detect.

To remove any influence of post-synaptic NMDARs, recordings were carried out where post-synaptic NMDARs were blocked using hyperpolarization ( $V_{\text {rest }}=-80 \mathrm{mV}$ ) and inclusion of MK-801 in the recording pipette. To reduce polysynaptic activity, experiments were carried out with high divalent ion concentrations in the circulating bath solution, increasing the likelihood for $\mathrm{Mg}^{2+}$ block of the NMDAR.

In control animals, under our recording conditions, there was little evidence for an influence of presynaptic NMDARs on evoked EPSCs at layer 4-2/3 synapses (Fig. 2A,D), consistent with prior work. On average, application of the NMDAR-antagonist APV had little effect on the PPR (baseline $0.89+0.06$ before versus APV $0.86+0.09 ; n=10$ cells and 7 mice), although the 
A

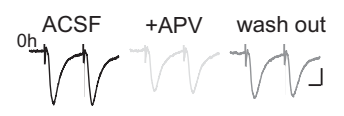

B
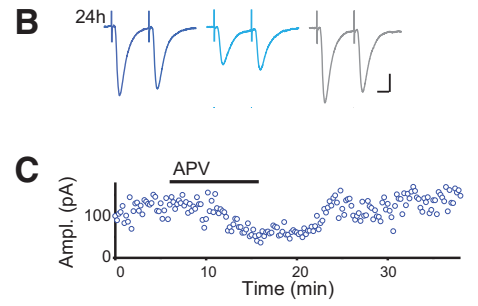

D

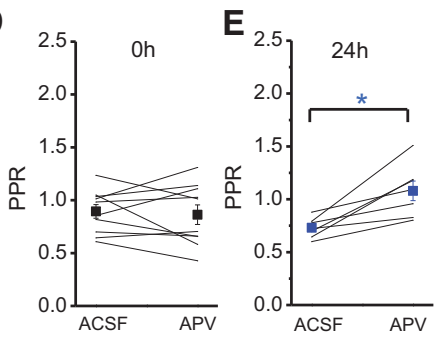

$\mathbf{F}$

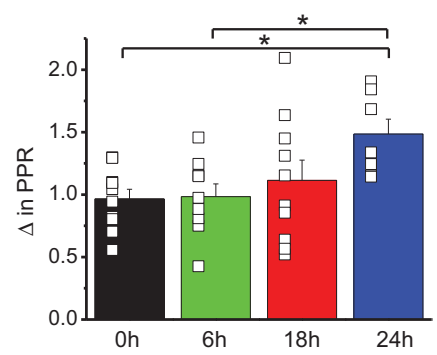

Figure 2. Presynaptic NMDARs at layer 4-2/3 synapses. (A) Average of 10 traces of evoked EPSCs before (black) after APV application (gray) and wash-out (light gray) in a control mouse. (B) Average evoked EPSC before (blue) after APV application (light blue) and wash-out (light gray) in a mouse after $24 \mathrm{~h}$ of SRE. (C) An example experiment showing the reduction of the first EPSCs amplitude after APV application in a mouse after 24 $\mathrm{h}$ of SRE. (D) The scatter plot of PPR from individual cells recorded at $0 \mathrm{~h}$ ( $n=10$ cells and 7 animals) before (ACSF) and after APV. (E) The same as in $D$ but at $24 \mathrm{~h}$ ( $n=7$ cells and 6 animals). $\left(^{*}\right) P=0.007$ paired $t$-test. $(F)$ Scatter plot of the change in PPR calculated by dividing the PPR of the baseline response/PPR after APV, averaged across each cell at $0 \mathrm{~h}(n=$ 10 cells and 7 animals), $6 \mathrm{~h}$ ( $n=9$ cells and 7 animals), $18 \mathrm{~h}(n=10$ cells and 8 animals), and $24 \mathrm{~h}$ ( $n=7$ cells and 6 animals) of SRE. $\left(^{*}\right)$ $P<0.05$ LMM.

effect on individual cells was variable. Importantly, low PPR values were not associated with a stronger APV effect, suggesting that this variance did not stem from heterogeneity under the influence of presynaptic NMDARs (Fig. 2D).

After $24 \mathrm{~h}$ of SRE, NMDAR-antagonist application significantly increased PPR values (Fig. 2B,C,E; $24 \mathrm{~h}$ SRE PPR baseline $0.73+0.04$ versus $24 \mathrm{~h} \mathrm{SRE}+$ APV $1.08+0.04 ; n=7$ cells in 6 mice; $P=0.007$ by paired $t$-test). This effect was reversed with washout of APV (Fig. 2B,C). The increase in PPR was especially notable in light of the fact that recording conditions were not modified to enhance detection of presynaptic NMDARs, as has been required in other studies (Brasier and Feldman 2008). The change in the ability of presynaptic NMDARs to facilitate release at layer $4-2 / 3$ synapses required at least $24 \mathrm{~h}$ of SRE to become apparent, and was not statistically significant with shorter durations of SRE (Fig. 2F). It was also independent of the recording temperature (Supplemental Fig. 2). In addition, the robustness of this result in the face of high levels of $\mathrm{Mg}^{2+}$ in the bath suggests that the presynaptic NMDARs involved may not be sensitive to $\mathrm{Mg}^{2+}$ block.

\section{NR2B-containing presynaptic NMDARs are not required to change PPR}

NMDARs are tetramers, composed of two NR1 subunits that combine with two NR2- or NR3 subunits to confer distinctive properties of $\mathrm{Mg}^{2+}$ blockade and decay kinetics (Monyer et al. 1994; Chatterton et al. 2002). The NR2B (GluN2B/Grin2b) subunit has been associated with long-term depression (Liu et al. 2004; Massey et al. 2004) but see Hrabetova et al. (2000) and Yoshimura et al. (2003), and activity-dependent up-regulation of presynaptic NR2B and NR3A (GluN3A/Grin3a) has been observed at some neocortical synapses (Yang et al. 2006; Larsen et al. 2014). Typically, NR2B is more prevalent during early development, and is replaced with NR2A in older animals. For example, in primary visual cortex, visual stimulation can trigger an increase in synaptic NR2A (Quinlan et al. 1999), and in somatosensory cortex post-synaptic NMDAR currents become progressive less sensitive to the NR2B-selective antagonist ifenprodil during post-natal development (Barth and Malenka 2001).

In our previous study, no changes in ifenprodil sensitivity of post-synaptic NMDAR-currents after $24 \mathrm{~h}$ of selective whisker experience were observed, suggesting that these receptors are not implicated in SRE metaplasticity (Clem et al. 2008). However, this analysis of post-synaptic NMDARs did not take into account the presence of potentially presynaptic NMDARs at layer 4-2/3 synapses.

First we examined the role of NR2B-containing NMDARs to the post-synaptic NMDAR-EPSC at multiple stages during SRE. As in previous studies, $24 \mathrm{~h}$ of SRE did not alter ifenprodil sensitivity (the fraction ifenprodil block of NMDA-EPSC at $0 \mathrm{~h}: 0.60 \pm$ $0.08,5$ cells in 5 mice, 24 h: $0.66 \pm 0.08,6$ cells in 3 mice, Fig. $3 \mathrm{~A}, \mathrm{~B})$. In contrast, ifenprodil sensitivity was significantly reduced at $18 \mathrm{~h}$ of SRE $(0.41 \pm 0.07,5$ cells in 5 mice, Fig. $3 \mathrm{~A}, \mathrm{~B})$. Interestingly, this shift was not associated with faster decay kinetics of the post-synaptic NMDAR-EPSC (Fig. 3C) either before or after ifenprodil application, despite the fact that NR2B-containing NMDARs have been shown to exhibit significantly longer decay kinetics (Monyer et al. 1994; Tovar and Westbrook 1999).

An alternative model to explain the decrease in ifenprodil block of the post-synaptic NMDAR-EPSC might be that presynaptic NMDARs are changing their subunit composition, replacing NR2B with another subunit. To determine whether this change in ifenprodil sensitivity might be linked to changes in putatively presynaptic NMDAR-subunit composition, we investigated the effect of ifenprodil on the PPR when post-synaptic NMDARs were blocked (Fig. 4). Ifenprodil significantly decrease the PPR in control animals, suggesting that presynaptic NR2B-containing NMDARs receptors facilitate release under basal conditions (0-h PPR baseline $0.94 \pm 0.09$ versus + ifenprodil $0.76 \pm 0.09 ; n=12$ cells in 7 mice; $P=0.029$ by paired $t$-test, Fig. 4A,C). However, ifenprodil failed to change the PPR at any stage after the onset of SRE (6-h PPR baseline $0.93 \pm 0.11$ versus + ifenprodil $1.05 \pm$ $0.19 ; n=9$ cells in 7 mice; $P=0.36$ by paired $t$-test and 18 -h PPR baseline $0.67 \pm 0.08$ versus + ifenprodil $0.77 \pm 0.12 ; n=13$ cells in 8 mice; $P=0.19$ by paired $t$-test Fig. $4 \mathrm{D}-\mathrm{F})$ - even at $24 \mathrm{~h}$ after SRE (24-h PPR baseline $0.61 \pm 0.04$ versus + ifenprodil $0.57 \pm 0.08 ; n=10$ cells in 6 mice; $P=0.063$ by paired $t$-test Fig. 4 B,D) when APV significantly enhanced the PPR (Fig. 2C-F). Thus, the NMDAR-dependent change in release probability initiated by SRE shown in Figure 2 cannot be attributed to a change in NR2B expression. If anything, the influence of NR2B-containing NMDARs on the PPR is reduced by SRE, since ifenprodil decreases 
A

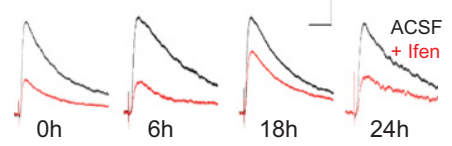

B

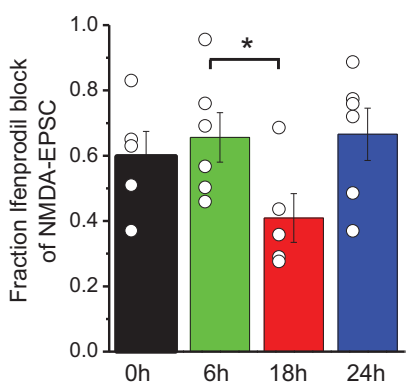

C

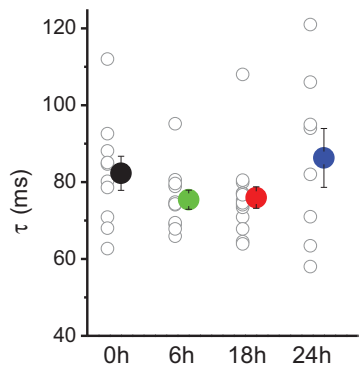

Figure 3. Ifenprodil sensitivity of post-synaptic NMDA currents decreases during the labile phase. (A) Example traces of evoked NMDA-EPSCs recorded at $+40 \mathrm{mV}$ at layer $4-2 / 3$ excitatory synapses before (black) and after ifenprodil application (red) at $0,6,18$, and $24 \mathrm{~h}$ after the onset of SRE. Calibration: $50 \mathrm{pA}, 50 \mathrm{msec}$. (B) Summary of the fractional block of NMDA-EPSC by ifenprodil at $0 \mathrm{~h}$ ( $n=5$ cells and 5 animals), $6 \mathrm{~h}$ ( $n=6$ cells and 6 animals), $18 \mathrm{~h}$ ( $n=5$ cells and 5 animals) and $24 \mathrm{~h}$ ( $n=6$ cells and 3 animals) of SRE. (C) Comparison of decay kinetics $(\tau)$ at $0 \mathrm{~h}$ ( $n=14$ cells and 5 animals), $6 \mathrm{~h}$ ( $n=11$ cells and 5 animals), 18 $\mathrm{h}(n=10$ cells and 3 animals), and $24 \mathrm{~h}$ ( $n=10$ cells and 3 animals) of SRE.

PPR in cells from control animals (Fig. 4C) and has no effect after $24 \mathrm{~h}$ of SRE (Fig. 4D).

\section{Whisker experience enhances the effect of presynaptic NMDARs on isolated EPSC amplitude}

Presynaptic NMDARs could influence release probability during bursting activity when presynaptic spikes can lead to persistent elevations in $\mathrm{Ca}^{2+}$ levels at the axon terminal (Bender et al. 2006b), but have also been shown to influence basal synaptic transmission. For example, APV decreases the amplitude of isolated, evoked EPSCs and can increase miniature EPSC (mEPSC) frequency in layer 2/3 neocortical neurons (Brasier and Feldman 2008; Larsen et al. 2011; Kunz et al. 2013). The effect of NMDARs on isolated EPSCs might be dissociable from their effects on PPR. Thus, we next investigated whether SRE might change the effect of presynaptic NMDARs on basal synaptic transmission at layer 4-2/3 synapses.

To isolate layer 4 inputs, we analyzed the effect of APV on evoked EPSCs at different time points after the onset of SRE. In control animals where post-synaptic NMDARs were blocked using post-synaptic hyperpolarization as well as inclusion of MK-801 in the post-synaptic recording pipette, APV modestly reduced EPSC amplitude (Fig. 5A,C; $16 \%$ reduction in EPSC amplitude, $n=10$ cells in 7 mice). As the duration of SRE increased, APV showed greater suppression of EPSC amplitude (Fig. 5A,C; 6 h 31\% decrease $n=9$ cells and 7 mice; $18 \mathrm{~h} 45 \%$ decrease $n=10$ cells and 8 mice; $24 \mathrm{~h}, 32 \%$ decrease $n=7$ cells and 6 mice). These data suggest that SRE triggers a change in presynaptic NMDARs that can regulate basal release properties.

To determine whether this shift in NMDAR-function might be attributable to an increase in functional expression of the NR2B subunit, we examined whether ifenprodil would have the same effect as the pan-NMDAR-antagonist APV (Fig. 5B,D). Ifenprodil had no effect on EPSC amplitude in cells from control animals ( $1.03 \%$ of baseline, $n=12$ cells and 7 animals), indicating that presynaptic NR2B-containing receptors play a negligible role under normal conditions. However, $6 \mathrm{~h}$ after the SRE onset, ifenprodil reduced EPSC amplitude by nearly half, a highly significant change (39\%, $n=9$ cell in 7 animals). This effect was apparently maintained at $18 \mathrm{~h}$ of SRE ( $30 \%, n=13$ cells in 8 animals), but had decreased by $24 \mathrm{~h}$ of SRE (13.5\%, $n=10$ cells in 7 animals). The effect of ifenprodil on basal transmission is notable, since it did not influence PPR at any time point tested (Fig. 4). These results dissociate the effect of these receptors on controlling the amplitude of single-evoked EPSCs from their effect on PPR. This finding is consistent with multiple, separate effects of presynaptic NMDARs at layer 4-2/3 synapses that are coordinately regulated by whisker activity.

How do NMDARs influence basal synaptic transmission? One hypothesis for how NMDARs facilitate basal synaptic transmission is that they are tonically active, gated by low levels of
A

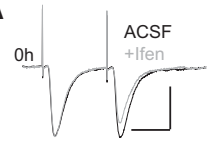

C 2
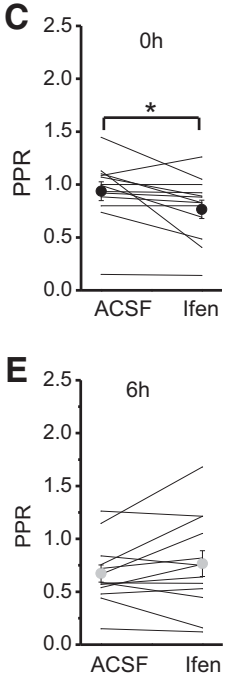
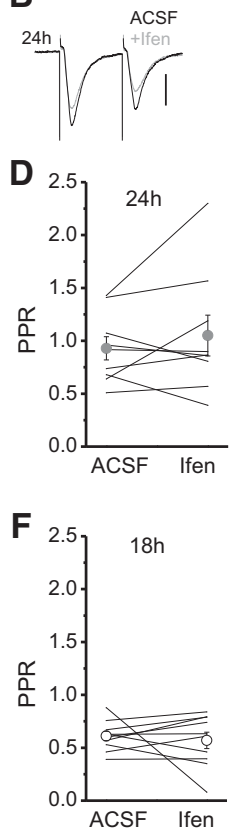

Figure 4. NR2B-containing presynaptic NMDARs are not required to change PPR. (A) Average traces of evoked EPSCs before (ACSF, black) and after ifenprodil application (gray) in a control mouse. Calibration: $50 \mathrm{pA}, 20 \mathrm{msec}$. (B) Average traces of evoked EPSCs before (black) and after ifenprodil (gray) in a mouse after $24 \mathrm{~h}$ of SRE. Calibration: $50 \mathrm{pA}$, 20 msec. (C) The scatter plot of PPR from individual cells at $0 \mathrm{~h}(n=12$ cells and 7 animals) before (ACSF) and after ifenprodil. $\left({ }^{*}\right) P=0.03$ paired $t$-test. $(D)$ The same as in C but recorded at $24 \mathrm{~h}$ of SRE $(n=10$ cells and 6 animals). (E) The same as in $C$ but recorded at $6 \mathrm{~h}$ of SRE ( $n=9$ cells and 7 animals). ( $F$ ) The same in $C$ but recorded at $18 \mathrm{~h}$ of SRE ( $n=13$ cells and 8 animals). 
A
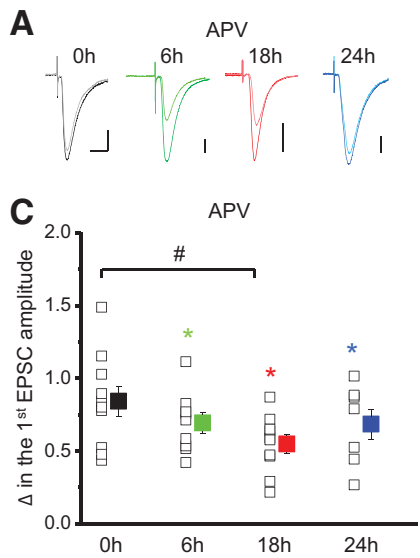

B
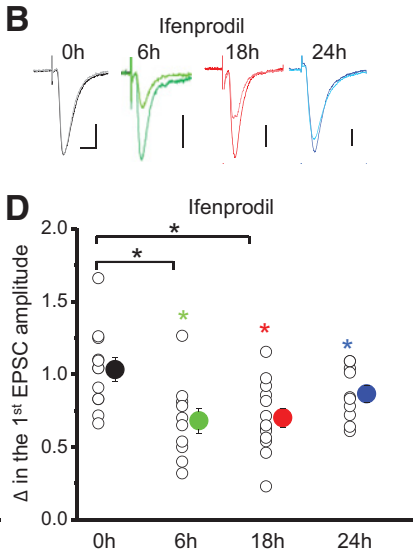

Figure 5. Presynaptic NMDARs enhance the amplitude of isolated EPSCs. (A) Example of average traces of evoked EPSCs before (dark colors) and after APV (light colors) at 0, 6, 18, and $24 \mathrm{~h}$ after the onset of SRE. (B) The same as in $A$ but before (dark colors) and after ifenprodil application (light colors). Calibration: $20 \mathrm{pA}, 10 \mathrm{msec}$. (C) Scatter plot of the mean change of EPSC amplitude after APV application recorded at $0 \mathrm{~h}$ ( $n=10$ cells and 7 animals), $6 \mathrm{~h}$ ( $n=9$ cells and 7 animals), $18 \mathrm{~h}$ ( $n=10$ cells and 8 animals) and $24 \mathrm{~h}$ ( $n=7$ cells and 6 animals) of SRE. Colored $\left({ }^{*}\right) P<0.5$ for the effect of drug, Wilcoxon rank test. $\left({ }^{\#}\right)$ $P<0.1 \mathrm{LMM}$ for the effect of SRE. $(D)$ The same as in $C$ but for ifenprodil application recorded at $0 \mathrm{~h}$ ( $n=12$ cells and 7 animals), $6 \mathrm{~h}$ ( $n=9$ cells and 7 animals), $18 \mathrm{~h}(n=13$ cells and 8 animals), and $24 \mathrm{~h}(n=10$ cells and 7 animals) of SRE. Colored $(*) P<0.05$ Wilcoxon rank test, for the effect of a drug, $(\#) P<0.05$ LMM for the effect of SRE.

ambient glutamate to depolarize the presynaptic cell and thus increase release probability (Sah et al. 1989; Shu et al. 2006). To determine whether whisker activation during SRE could alter this property, we examined the effect of APV on resting membrane potential in layer 4 neurons.

In control animals, APV resulted in a $4 \mathrm{mV}$ hyperpolarizing shift in $V_{\text {rest }}$ (Fig. 6A-C; $4.04+1.4 \mathrm{mV}$ shift; $n=8$ cells in 4 mice). The effect of APV was highly variable from cell to cell (range 8.4-2.4-mV hyperpolarization; Fig. 6C), a property that makes it difficult to detect statistically significant changes without a very large number of recordings. Interestingly, ifenprodil showed no effect on $V_{\text {rest }}$ in control animals (Fig. 6C; $0.15+1.3$ $\mathrm{mV}$ shift; $n=6$ cells in 3 mice). This indicates that NR2B-lacking NMDARs are responsible for depolarizing layer 4 neurons. Although they can influence EPSC amplitude, NR2B-containing NMDARs do not contribute to resting membrane potential in layer 4 neurons under our recording conditions.

We hypothesized that whisker experience might lead to a larger role for NMDARs in setting resting membrane potential, such that these receptors might provide a tonic current that depolarizes layer 4 neurons and increase release probability. However, after $24 \mathrm{~h}$ of SRE, we observed no change in APV-dependent depolarization of layer 4 neurons compared with control (Fig. 6C). This time point was when APV had a maximal effect on PPR and also could reduce evoked EPSC amplitude. Thus, these data dissociate the effect of NMDARs on $V_{\text {rest }}$ from their influence on the release properties of glutamatergic synapses.

Some studies have identified a role for NMDARs in regulating miniature EPSC frequency (but not amplitude Kunz et al. 2013), consistent with a presynaptic effect for these receptors. However, under our recording conditions, we observed no significant reduction in mEPSC frequency with APV in control $(101+4.6 \%, n=4$ cells in 2 mice) or after SRE ( $93+9.6 \%, n=6$ cells in 2 mice; Supplemental Fig. 3). At warmer recording temperatures, we did observe an SRE-dependent increase in the effect of NMDARs on
mEPSC frequency (control 91.8 $+2.9 \%, n=8$ cells in 4 mice versus SRE $79.7+4.1 \%, n=8$ cells in 6 mice). Overall, these data are consistent with a SRE-dependent increase in presynaptic NMDAR function.

\section{Discussion}

Here we show that enhanced sensory input, using a protocol for retaining only a single row of whiskers on both sides of the mouse face, can enhance synaptic transmission through at least two different presynaptic mechanisms. First, synaptic release during high-frequency stimulation shifts to become significantly more depressing compared with control conditions, and this increase is at least partly due to APV-sensitive, non-NR2B-containing, NMDARs. Second, we detected a significant increase in the contribution of presynaptic NMDARs in increasing the amplitude of isolated EPSCs. In control animals, presynaptic NMDARs play a negligible role in modulating basal EPSC amplitude. However, shortly after SRE begins (between 6 and $18 \mathrm{~h}$ after the onset); presynaptic NMDARs enhance evoked EPSC-amplitude at layer 4-2/3 synapses. These release-enhancing NMDARs are likely to contain NR2B, since the AMPAR-EPSC was suppressed with both APV and also the NR2B-selective antagonist ifenprodil. These data suggest that both NR2B-containing and NR2B-lacking NMDARs are regulated during experience-dependent plasticity at layer 4-2/3 synapses in the developing barrel cortex.

\section{SRE changes presynaptic properties: enhancing release}

There has been a great deal of interest in how alterations in sensory experience shift neural firing and synaptic properties. However, it is easier to analyze how sensory deprivation changes circuit function (Lendvai et al. 2000; Bender et al. 2006a; Jiao et al. 2006), rather than studying the effect of enhanced sensory activity since this requires identification of a precise neocortical region that represents the activated sensory input. For example, in the visual cortex, intermingling of left- and right-eye driven cells within the binocular zone makes it difficult to isolate specific changes that are related to the spared sensory input. Using the anatomical map of the peripheral whiskers represented in the barrel field, we were able to precisely target the barrel columns
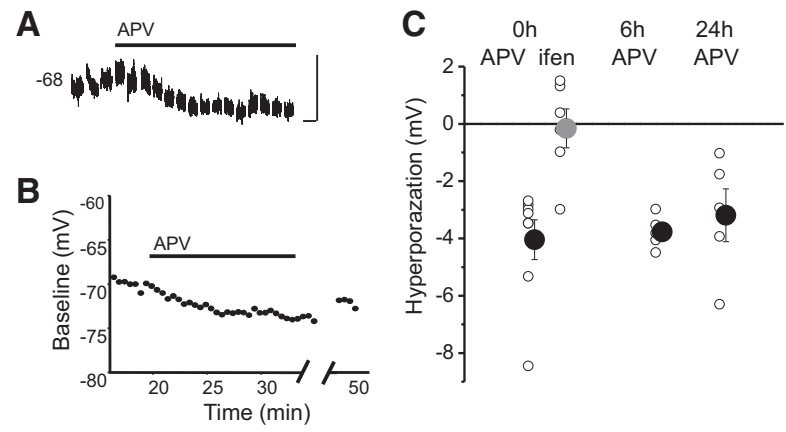

Figure 6. Tonic activation of non-NR2B-NMDA receptors contributes to the resting membrane potential in layer 4 excitatory neurons. (A) Example traces of membrane potential recorded with APV application. To diminish data points for the figure every short trace shows $30 \mathrm{sec}$ of recording truncated every $1 \mathrm{~min}$. Calibration: $10 \mathrm{mV}, 30 \mathrm{sec}$. (B) Example of the change of the membrane potential from the same cell shown above. (C) Mean hyperpolarization of the membrane potential after APV application at $0 \mathrm{~h}$ ( $n=8$ cells and 4 animals), $6 \mathrm{~h}$ ( $n=8$ cells and 4 animals), and $24 \mathrm{~h}$ ( $n=5$ cells and 3 animals) of SRE. Gray circle shows the effect of ifenprodil application on the membrane potential at $0 \mathrm{~h}$ only ( $n=6$ cells and 3 animals). 
corresponding to the spared whisker in order to investigate how presynaptic function could be altered during stimulation-induced plasticity.

Previous studies have shown that layer $4-2 / 3$ synapses in sensory-deprived neocortical regions become facilitating (i.e., an increase in PPR) after sensory deprivation, suggesting that release probability is decreased in the absence of the sensory input (Bender et al. 2006a). In contrast, we observed an increase in release probability in the spared sensory representation: synapses become more depressed and PPR is reduced. At a population level across many layer 4 neurons, these findings suggest that weaker whisker stimuli may be able to more effectively evoke a response in layer $2 / 3$ neurons, increasing overall EPSC amplitude so that post-synaptic spikes in layer $2 / 3$ neurons are generated with greater frequency. In addition, it remains possible that other postsynaptic mechanisms, such as altered receptor desensitization, may be altered by SRE and could contribute to the apparent change in release measured by PPR. However, our results are consistent with other studies that show presynaptic NMDARs can regulate release, and are regulated by sensory-evoked activity (Brasier and Feldman 2008; Rodríguez-Moreno and Paulsen 2008; Kunz et al. 2013; Larsen et al. 2014).

Interestingly, increased release probability has also been described in other preparations where overall levels of activity are increased, after chemically induced seizures or in cortical dysplasia (Scimemi et al. 2006; Yang et al. 2006; Chen et al. 2007; Upreti et al. 2012) but see Pitsch et al. (2012). The changes that are reported here are anti-homeostatic; they do not act to dampen the excitability of neurons in the face of increased sensory-evoked activity. This contrasts to other studies that have investigated the effect of decreased neural activity that enhances release probability (Bacci et al. 2001; Murthy et al. 2001; Desai et al. 2002; Yashiro and Philpot 2008; Lazarevic et al. 2013); it may be that homeostatic adjustments in release are engaged by decreased but not increased sensory-evoked activity, and are not fully bidirectional.

\section{SRE induces both pre- and post-synaptic changes in NMDAR subunit composition}

Our findings demonstrate that there is a change in the contribution of presynaptic NMDARs in mediating presynaptic release at layer $4-2 / 3$ synapses. Are there also changes in post-synaptic NMDARs? Based upon data in Figures 3 and 4, we propose that SRE leads to a change in both post-synaptic and presynaptic NR2B-containing NMDARS. Under control conditions, bath application of the NR2B-specific antagonist ifenprodil blocks specifically the post-synaptic NMDAR-EPSC by $60 \%$ (Fig. 3) without influencing presynaptic release (Fig. 4).

However, $6 \mathrm{~h}$ after the SRE onset, presynaptic NR2B-containing NMDARs show a new role in facilitating EPSC amplitude (Fig. 4). Because we know that these NR2B receptors are presynaptic, and yet ifenprodil application still reduces the post-synaptic NMDAR-EPSC by a comparable amount compared with control (Fig. 3), this suggests that there is in fact a decrease in the contribution of post-synaptic NR2B-containing NMDARs.

In $18 \mathrm{~h}$ after SRE, presynaptic, NR2B-containing NMDARs still show a significant influence on enhancing the amplitude of layer 4-2/3 AMPAR-mediated EPSCs, since ifenprodil application reduces EPSC amplitude by $30 \%$ (Fig. 4). At the same time point, ifenprodil also decrease the post-synaptic NMDAR-EPSC by $41 \%$ (Fig. 3), an effect that must be the sum of both pre- and postsynaptic effects. Taken together, these data suggest that the entire ifenprodil-mediated inhibition on the post-synaptic NMDAREPSC might be mediated almost entirely through presynaptic NMDARs, which robustly enhance release at this time point. Thus, sensory experience can regulate both post-synaptic and pre- synaptic NR2B: NR2B-containing NMDARs are down-regulated in the post-synaptic compartment, and NR2B-containing NMDARs are functionally up-regulated at presynaptic sites. These probably include the presynaptic compartment but may also be localized at other sites with the layer 4 neuron or in glial cells, which also express NMDARs (Salter and Fern 2005; Lee et al. 2010; Zhou et al. 2010; Dzamba et al. 2013). The mechanisms by which glialcell NR2B can regulate presynaptic release have not been well described.

Previous studies have suggested that NR2A levels can be positively regulated by activity levels, changing the relative NR2A/B ratio. Interestingly, in these biochemical studies of isolated synaptoneurosomes, the levels of NR2B were not found to be altered (Quinlan et al. 1999; Philpot et al. 2001). It may be that previous studies were not able to detect overall changes in NR2B levels because a post-synaptic decrease was offset by presynaptic increase. However, the specific cellular location of SRE-regulated NR2B subunits remains unknown.

Presynaptic NMDARs have been shown to contain NR3A, a glycine-binding subunit that confers unique electrophysiological properties on NMDARs (Larsen et al. 2011). In addition, the presence of NR3A can reduce APV binding in some NMDAR subtypes (Chatterton et al. 2002). We identified a discrepancy in the effect of APV (which did not change PPR in control animals) and ifenprodil (which slightly decreased PPR in control animals). This might be due to a predominance of NR3A-containing NMDARs in control animals that reduce APV efficacy. The ifenprodil sensitivity of these receptors suggests that they contain NR2B. Future experiments to dissect the role of subunit composition on drug sensitivity, as well as to identify the specific subunit composition of presynaptic NMDARs at developing layer 4-2/3 synapses, will be required to test this possibility.

\section{Presynaptic NMDARs limit experience-dependent synaptic potentiation}

There is strong experimental evidence for presynaptic NMDARs at layer 4-2/3 synapses. In somatosensory cortex, dual whole-cell recording experiments show that pharmacological blockade of presynaptic NMDARs can prevent the induction of t-LTD, and that this form of synaptic plasticity is developmentally regulated (Rodríguez-Moreno and Paulsen 2008; Banerjee et al. 2009; Rodríguez-Moreno et al. 2011; Itami and Kimura 2012;). These presynaptic NMDARs are critical for synaptic depression at developing layer $4-2 / 3$ synapses and are not found at horizontal, layer $2 / 3-2 / 3$ synapses in the barrel cortex. Synapse-specific presynaptic NMDARs that play a critical role in depression have also been observed at other neocortical synapses, including layer 5 (Buchanan et al. 2012).

As presynaptic NMDARs are tied to the induction of t-LTD, it is tempting to speculate that an increase in presynaptic NMDARs during SRE might make it more likely that synaptic depression will be induced at layer 4-2/3 synapses. Interestingly, the increase of presynaptic NMDAR function occurs around the same time as the labile phase of SRE-induced synaptic plasticity, where NMDAR activation is associated with a reduction in EPSC amplitude at layer 4-2/3 synapses (Clem et al. 2008; Wen and Barth 2012; Wen et al. 2013). Previous studies that identified this phase of NMDAR-dependent depression during SRE did not selectively block presynaptic NMDARs (and thus cannot conclude that the experience-dependent change in NMDAR properties is localized to this synaptic compartment). Such a mechanism might be a way to shift plasticity-induction thresholds to favor LTD over LTP induction (Abraham and Bear 1996), a phenomenon that has previously been described in this system (Clem et al. 2008). 
Activity-dependent regulation of presynaptic NMDARs may serve as a metaplastic switch to limit the degree of synaptic potentiation that can occur at layer 4-2/3 synapses, maintaining these inputs within a restricted range. Because the labile phase is transient (Wen et al. 2013), we predict that at longer durations of SRE the increased role of presynaptic NMDARs will revert back to baseline levels, during the stabilization phase. It remains unknown what are the precise events required to trigger a change in presynaptic NMDARs: whether post-synaptic potentiation and retrograde signaling to the layer 4 presynaptic terminal is required, or whether the sustained activity of presynaptic neurons may be sufficient to regulate these NMDARs.

\section{Subunit composition of presynaptic NMDARs that are regulated by sensory experience}

Our results suggest that presynaptic NMDARs may consist of two distinct types, each with a different role in regulating presynaptic release. Presynaptic NMDARs that are blocked by APV act to decrease the PPR, suggesting that these receptors facilitate glutamate release. This effect was not observed with the NR2B-selective antagonist, indicating that the subset of receptors involved in the PPR effect lack NR2B. Because our experiments were carried out using high levels of $\mathrm{Mg}^{2+}$ in the circulating bath, we propose that these presynaptic receptors are likely to contain the $\mathrm{Mg}^{2+}$ insensitive subunits NR2C, NR2D, or NR3A. Previous studies have shown that layer 4 neurons express NR2C (Binshtok et al. 2006; Banerjee et al. 2009) and NR3A, the latter of which is observed presynaptically by immunoEM (Larsen et al. 2011).

In contrast to the NMDARs involved in regulating the PPR, we also found that NMDARs can enhance the amplitude of isolated EPSCs. This effect was not observed in control animals, but was significant within $6 \mathrm{~h}$ of the SRE onset. In these experiments, the effect of APV was nearly identical to ifenprodil, indicating that this subset of presynaptic NMDARs contains the NR2B subunit. This is consistent with previous studies that have suggested presynaptic NMDARs in visual cortex contain both NR3A as well as the NR2B subunit (Larsen et al. 2011).

Interestingly, NR3A levels sharply increase during development and then are reduced during the third post-natal week in mice, and it has been proposed that this subunit might be related to the developmental regulation of plasticity (Kehoe et al. 2013). It is possible that SRE induces an up-regulation of NR3A in layer 4 neurons, consistent with their resistance to Mg-block. Because t-LTD is absent in NR3A knockout mice, it appears that NR3A is required for t-LTD (Larsen et al. 2011). The activity-dependent up-regulation of this subunit may facilitate t-LTD at synapses strengthened by SRE.

\section{Conclusion}

Here we show that experience-dependent increases in the sensoryevoked output of neurons in primary somatosensory cortex may be mediated in part by increased presynaptic release probability at layer 4-2/3 synapses. Because NR2B-lacking NMDARs influence the PPR as well as the amplitude of isolated evoked EPSCs, and NR2B-containing NMDARs only influence the amplitude of isolated evoked EPSCs, we predict that they are localized to different compartments within the layer $4-2 / 3$ subcircuit.

The timing of these changes in putatively presynaptic NMDARs occurs roughly in parallel with changes in the susceptibility of layer 4-2/3 synapses in the spared barrel column to undergo synaptic depression. Our previous studies have shown that sensory-induced plasticity is initiated by an NMDAR-dependent form of synaptic strengthening, but that after the first $12 \mathrm{~h}$ of altered input, NMDAR-activation now leads to a weakening of strengthened synapses, where depression is favored over potentiation. Our findings suggest that this transition, from the initiation to the labile phase of experience-dependent plasticity, may occur because of an up-regulation of presynaptic NMDARs, a pathway that has been shown to activate t-LTD at layer $4-2 / 3$ synapses.

\section{Materials and Methods}

\section{Animals}

Wild type C57BL6 mice were used for experiments at the age of P12-P14, of either sex. In a few cases ( $<10 \%$ of experiments), nontransgenic animals from a colony backcrossed 12-18 generations into the C57BL6 strain (Harlan) were used. Because these animals did not carry any transgene, they were considered functionally equivalent to wild-type mice. In the single-row experience (SRE) paradigm, all whiskers were deprived bilaterally except a single set of D-row whiskers on the right side (Wen et al. 2013). Animals were returned to their home cage for a variable amount of time $(0,6,18$, and $24 \mathrm{~h})$ before electrophysiological recordings. Control animals were whisker-intact littermates of deprived animals from the same age range (P12-P14). Whiskers were not directly stimulated by the experimenter.

\section{Slice preparation and whole-cell recording}

Animals were anesthetized with isofluorane, decapitated and prepared in cold artificial CSF (ACSF) at $2{ }^{\circ} \mathrm{C}-6^{\circ} \mathrm{C}$ composed of the following (mM): $119 \mathrm{NaCl}, 2.5 \mathrm{KCl}, 2.5 \mathrm{CaCl}_{2}, 1.3 \mathrm{MgSO}_{4}, 1$ $\mathrm{NaH}_{2} \mathrm{PO}_{4}, 26.2 \mathrm{NaHCO}_{3}$, and 11 glucose and equilibrated with $95 / 5 \% \mathrm{O}_{2} / \mathrm{CO}_{2}$. Coronal slices with $350-\mu \mathrm{m}$ thickness were vibratome-sectioned by an "across-row" protocol. The hemisphere related to the spared whiskers was sectioned along a $45^{\circ}$ plane toward the midline (Finnerty et al. 1999) to obtain slices with five barrels where every barrel belongs to one row. The barrel column representing the spared D whiskers was targeted for recording. Slices were maintained and whole-cell recordings were performed at room temperature $\left(24^{\circ} \mathrm{C}\right)$ unless otherwise indicated. Somata of lower layer $2 / 3$ pyramidal neurons or layer 4 excitatory neurons in barrel cortex were targeted for whole-cell recording with borosilicate glass electrodes with a resistance of 4-8 M $\Omega$. Pyramidal cell identity was confirmed after the recording session by pyramidal somata morphology and the presence of apical dendrite and dendritic spines. Layer 4 excitatory neurons were recognized by the presence of dendritic spines. To record evoked EPSC in layer $2 / 3$ electrode internal solution was composed of the following (in $\mathrm{mM}$ ): 130 cesium gluconate, 10 HEPES, 0.5 EGTA, $8 \mathrm{NaCl}$, 10 tetraethylammonium chloride, $4 \mathrm{Mg}$-ATP, and 0.4 Na-GTP, 5 QX-314, and 1 MK-801 at pH 7.25-7.30, 290-300 mOsm and contained trace amounts of Alexa 568.

To record spontaneous activity of layer 4 neurons electrode internal solution was composed of the following (in $\mathrm{mM}$ ): 116 potassium gluconate, $6 \mathrm{KCl}, 8 \mathrm{NaCl}, 0.5 \mathrm{EGTA}, 4 \mathrm{MgATP}$, and 0.4 NaGTP, at pH 7.25-7.35, 290 mOsm and contained trace amounts of Alexa 568 .

Only cells with $R$ series $\leq 30 \mathrm{M} \Omega$ and $R$ input $\geq 200 \mathrm{M} \Omega$, where changes in either measurement were $>30 \%$, were included for analysis. Electrophysiological data were acquired by Multiclamp 700A (Molecular Devices) and a National Instruments acquisition interface. The data were filtered at $3 \mathrm{kHz}$ and digitized at $10 \mathrm{kHz}$ and collected by Igor Pro 6.0 (Wavemetrics). Extracellular simulation was controlled by a Master-8 (A.M.P.I.) and a stimulus isolator Isoflex (A.M.P.I.). Stimulation of presynaptic afferents with ISI of $33 \mathrm{msec}$ at $0.1 \mathrm{~Hz}$ was performed using a glass monopolar electrode filled with ACSF and placed in the center of the barrel in layer 4. Evoked EPSCs were elicited with 3-10 $\mu \mathrm{A}$ of current in order to yield an EPSC $\sim 100 \mathrm{pA}$. To record the effect of NMDA receptors on resting membrane potential, layer 4 neurons activity was recorded in current clamp mode and the baseline shift was calculated after APV application. 


\section{NMDAR-mediated EPSC and ifenprodil sensitivity} Evoked NMDAR-mediated EPSCs were isolated by including 1,2,3,4-tetrahydro-6-nitro-2,3-dioxo-benzo[ $f$ ]quinoxaline-7-sulfonamide hydrate (NBQX, $5 \mu \mathrm{M}$ ) and picrotoxin (Ptx; $50 \mu \mathrm{M})$ in the bath solution (regular ACSF). Layer $2 / 3$ pyramidal cells were voltage-clamped at $+40 \mathrm{mV}$. A single-exponential function was fitted to the average NMDA-EPSC trace from its peak to 200 msec after the stimulus onset and a decay constant $\tau$ was determined from the fit. Baseline recordings of NMDA currents lasted at least $10 \mathrm{~min}$ to make sure there is a stable baseline of $5-10$ min. To assess the content of NR2B-containing NMDARs, ifenprodil $(5 \mu \mathrm{M})$, a specific NR2B antagonist was infused to the recording chamber locally while recordings were continued. Because the action of ifenprodil was poorly reversible (Kumar and Huguenard 2003), we expected no recovery of the currents after drug application which was observed in all of our experiments. The average baseline response was 125 obtained by averaging $20-30$ sweeps right before the drug was added. The average post-drug response was obtained by averaging 20-30 sweeps toward the end of recording after the response stabilized.

\section{Measurement of presynaptic NMDARs}

Evoked AMPAR-mediated EPSC (AMPA-EPSC) was isolated by including Ptx $(50 \mu \mathrm{M})$ in the bath solution containing $4 \mathrm{mM} \mathrm{Ca}^{2+}$ and $4 \mathrm{mM} \mathrm{Mg}^{2+}$ to block polysynaptic activity. Layer $2 / 3$ pyramidal cells were voltage-clamped at $-80 \mathrm{mV}$ while post-synaptic NMDARs were blocked by noncompetitive open-channel blocker of NMDARs, $(+)$-5-methyl-10,11-dihydro-5H-dibenzo[a, $d]$ cyclohepten-5,10-imine maleate, MK801 (1 mM) included in the internal solution (Kumar and Huguenard 2003). Baseline recordings lasted $\sim 10 \mathrm{~min}$ to allow efficient blockade of post-synaptic NMDARs by MK801. Two pulses with an interpulse interval of $33 \mathrm{msec}(30 \mathrm{~Hz})$ (Brasier and Feldman 2008) were administered at a frequency of $0.1 \mathrm{~Hz}$ for recordings of paired-pulse ratio of AMPA-EPSCs. Cells with a latency of 2-5 msec for the first EPSC and that showed clear monosynaptic component EPSC were included for analysis. Baseline responses and post-drug responses were obtained by averaging 10 sweeps right before drug application or 5-10 min after drug application when responses were normally stabilized. PPR expressed as the ratio of the second EPSC amplitude and the first EPSC amplitude of the average traces (second EPSC/first EPSC).

\section{Statistics}

Typically, only one to two slices per brain contained the entire complement of five barrels. Not more than two cells from one mouse were recorded and included for the analysis, with one exception where three cells from one control mouse were collected.

Statistical significance was calculated between groups for each timepoint or drug manipulation. Comparison between groups of mice were performed by one-way nonparametric ANOVA using number of cells, or to accommodate random effects caused by animal-to-animal variability in our data sets, a generalized linear mixed model (LMM) followed by simultaneous multiple pairwise comparisons. The $P$ values were adjusted for multiple comparisons described by Hochberg and Benjamini (1990). The effect of a drug on the response within one group was tested by paired $t$-test on cells. Statistical analyses were performed using the software program R or InStat (demo version).

\section{Acknowledgments}

This work was supported by NIH DA0171-88. We thank Joanne Steinmiller for expert animal care, members of the Barth laboratory and Daniel J. Brasier for helpful comments and critical reading of the manuscript.

\section{References}

Abraham WC, Bear MF. 1996. Metaplasticity: the plasticity of synaptic plasticity. Trends Neurosci 19: 126-130.
Aoki C, Venkatesan C, Go CG, Mong JA, Dawson TM. 1994. Cellular and subcellular localization of NMDA-R1 subunit immunoreactivity in the visual cortex of adult and neonatal rats. J Neurosci 14: 5202-5222.

Bacci A, Coco S, Pravettoni E, Schenk U, Armano S, Frassoni C, Verderio C, De Camilli P, Matteoli M. 2001. Chronic blockade of glutamate receptors enhances presynaptic release and downregulates the interaction between synaptophysin-synaptobrevin-vesicle-associated membrane protein 2. J Neurosci 21: 6588-6596.

Banerjee A, Meredith RM, Rodriguez-Moreno A, Mierau SB, Auberson YP, Paulsen O. 2009. Double dissociation of spike timing-dependent potentiation and depression by subunit-preferring NMDA receptor antagonists in mouse barrel cortex. Cereb Cortex 19: 2959-2969.

Barth AL, Malenka RC. 2001. NMDAR EPSC kinetics do not regulate the critical period for LTP at thalamocortical synapses. Nat Neurosci 4: $235-236$.

Barth AL, Gerkin RC, Dean KL. 2004. Alteration of neuronal firing properties after in vivo experience in a FosGFP transgenic mouse. J Neurosci 24: 6466-6475.

Bender KJ, Allen CB, Bender VA, Feldman DE. 2006a. Synaptic basis for whisker deprivation-induced synaptic depression in rat somatosensory cortex. J Neurosci 26: 4155-4165.

Bender VA, Bender KJ, Brasier DJ, Feldman DE. 2006b. Two coincidence detectors for spike timing-dependent plasticity in somatosensory cortex. J Neurosci 26: 4166-4177.

Berretta N, Jones RS. 1996. Tonic facilitation of glutamate release by presynaptic $N$-methyl-D-aspartate autoreceptors in the entorhinal cortex. Neuroscience 75: 339-344.

Binshtok AM, Fleidervish IA, Sprengel R, Gutnick MJ. 2006. NMDA receptors in layer 4 spiny stellate cells of the mouse barrel cortex contain the NR2C subunit. I Neurosci 26: 708-715.

Brasier DJ, Feldman DE. 2008. Synapse-specific expression of functional presynaptic NMDA receptors in rat somatosensory cortex. J Neurosci 28: 2199-2211.

Buchanan KA, Blackman AV, Moreau AW, Elgar D, Costa RP, Lalanne T, Tudor Jones AA, Oyrer J, Sjöström PJ. 2012. Target-specific expression of presynaptic NMDA receptors in neocortical microcircuits. Neuron 75: 451-466.

Charton JP, Herkert M, Becker CM, Schröder H. 1999. Cellular and subcellular localization of the 2B-subunit of the NMDA receptor in the adult rat telencephalon. Brain Res 816: 609-617.

Chatterton JE, Awobuluyi M, Premkumar LS, Takahashi H, Talantova M, Shin Y, Cui J, Tu S, Sevarino KA, Nakanishi N, et al. 2002. Excitatory glycine receptors containing the NR3 family of NMDA receptor subunits. Nature 415: 793-798.

Cheetham CE, Hammond MS, Edwards CE, Finnerty GT. 2007. Sensory experience alters cortical connectivity and synaptic function site specifically. J Neurosci 27: 3456-3465.

Chen HX, Xiang H, Roper SN. 2007. Impaired developmental switch of short-term plasticity in pyramidal cells of dysplastic cortex. Epilepsia 48: $141-148$.

Clem RL, Barth A. 2006. Pathway-specific trafficking of native AMPARs by in vivo experience. Neuron 49: 663-670.

Clem RL, Celikel T, Barth AL. 2008. Ongoing in vivo experience triggers synaptic metaplasticity in the neocortex. Science 319: $101-104$.

Clem RL, Anggono V, Huganir RL. 2010. PICK1 regulates incorporation of calcium-permeable AMPA receptors during cortical synaptic strengthening. J Neurosci 30: 6360-6366.

Corlew R, Wang Y, Ghermazien H, Erisir A, Philpot BD. 2007. Developmental switch in the contribution of presynaptic and postsynaptic NMDA receptors to long-term depression. J Neurosci 27: $9835-9845$

Corlew R, Brasier DJ, Feldman DE, Philpot BD. 2008. Presynaptic NMDA receptors: newly appreciated roles in cortical synaptic function and plasticity. Neuroscientist 14: 609-625.

Desai NS, Cudmore RH, Nelson SB, Turrigiano GG. 2002. Critical periods for experience-dependent synaptic scaling in visual cortex. Nat Neurosci 5: $783-789$.

Di Cristo G, Chattopadhyaya B, Kuhlman SJ, Fu Y, Bélanger MC, Wu CZ, Rutishauser U, Maffei L, Huang ZJ. 2007. Activity-dependent PSA expression regulates inhibitory maturation and onset of critical period plasticity. Nat Neurosci 10: 1569-1577.

Dzamba D, Honsa P, Anderova M. 2013. NMDA receptors in glial cells: pending questions. Curr Neuropharmacol 11: 250-262.

Feldman DE, Nicoll RA, Malenka RC, Isaac JT. 1998. Long-term depression at thalamocortical synapses in developing rat somatosensory cortex. Neuron 21: $347-357$.

Finnerty GT, Roberts LS, Connors BW. 1999. Sensory experience modifies the short-term dynamics of neocortical synapses. Nature 400: $367-371$. 
Gu Y, Huang S, Chang MC, Worley P, Kirkwood A, Quinlan EM. 2013. Obligatory role for the immediate early gene NARP in critical period plasticity. Neuron 79: 335-346.

Hensch TK. 2005. Critical period plasticity in local cortical circuits. Nat Rev Neurosci 6: 877-888.

Hochberg Y, Benjamini Y. 1990. More powerful procedures for multiple significance testing. Stat Med 9: 811-818.

Hrabetova S, Serrano P, Blace N, Tse HW, Skifter DA, Jane DE, Monaghan DT, Sacktor TC. 2000. Distinct NMDA receptor subpopulations contribute to long-term potentiation and long-term depression induction. J Neurosci 20: RC81.

Itami C, Kimura F. 2012. Developmental switch in spike timing-dependent plasticity at layers $4-2 / 3$ in the rodent barrel cortex. J Neurosci 32: 15000-15011.

Jacob V, Petreanu L, Wright N, Svoboda K, Fox K. 2012. Regular spiking and intrinsic bursting pyramidal cells show orthogonal forms of experience-dependent plasticity in layer V of barrel cortex. Neuron 73: 391-404.

Jiao Y, Zhang C, Yanagawa Y, Sun QQ. 2006. Major effects of sensory experiences on the neocortical inhibitory circuits. J Neurosci 26: $8691-8701$.

Kehoe LA, Bernardinelli Y, Muller D. 2013. GluN3A: an NMDA receptor subunit with exquisite properties and functions. Neural Plast 2013: 145387.

Knott GW, Quairiaux C, Genoud C, Welker E. 2002. Formation of dendritic spines with GABAergic synapses induced by whisker stimulation in adult mice. Neuron 34: $265-273$.

Kuhlman SJ, Olivas ND, Tring E, Ikrar T, Xu X, Trachtenberg JT. 2013. A disinhibitory microcircuit initiates critical-period plasticity in the visual cortex. Nature 501: 543-546.

Kumar SS, Huguenard JR. 2003. Pathway-specific differences in subunit composition of synaptic NMDA receptors on pyramidal neurons in neocortex. J Neurosci 23: 10074-10083.

Kunz PA, Roberts AC, Philpot BD. 2013. Presynaptic NMDA receptor mechanisms for enhancing spontaneous neurotransmitter release. J Neurosci 33: $7762-7769$.

Larsen RS, Corlew RJ, Henson MA, Roberts AC, Mishina M, Watanabe M, Lipton SA, Nakanishi N, Pérez-Otaño I, Weinberg RJ, et al. 2011. NR3A-containing NMDARs promote neurotransmitter release and spike timing-dependent plasticity. Nat Neurosci 14: 338-344.

Larsen RS, Smith IT, Miriyala J, Han JE, Corlew RJ, Smith SL, Philpot BD. 2014. Synapse-specific control of experience-dependent plasticity by presynaptic NMDA receptors. Neuron 83: 879-893.

Lazarevic V, Pothula S, Andres-Alonso M, Fejtova A. 2013. Molecular mechanisms driving homeostatic plasticity of neurotransmitter release. Front Cell Neurosci 7: 244

Lee MC, Ting KK, Adams S, Brew BJ, Chung R, Guillemin GJ. 2010. Characterisation of the expression of NMDA receptors in human astrocytes. PLoS One 5: e14123.

Lendvai B, Stern EA, Chen B, Svoboda K. 2000. Experience-dependent plasticity of dendritic spines in the developing rat barrel cortex in vivo. Nature 404: 876-881.

Liu L, Wong TP, Pozza MF, Lingenhoehl K, Wang Y, Sheng M, Auberson YP, Wang YT. 2004. Role of NMDA receptor subtypes in governing the direction of hippocampal synaptic plasticity. Science 304: $1021-1024$.

Massey PV, Johnson BE, Moult PR, Auberson YP, Brown MW, Molnar E, Collingridge GL, Bashir ZI. 2004. Differential roles of NR2A and NR2B-containing NMDA receptors in cortical long-term potentiation and long-term depression. J Neurosci 24: 7821-7828.

Monyer H, Burnashev N, Laurie DJ, Sakmann B, Seeburg PH. 1994. Developmental and regional expression in the rat brain and functional properties of four NMDA receptors. Neuron 12: 529-540.

Morishita H, Miwa JM, Heintz N, Hensch TK. 2010. Lynx1, a cholinergic brake, limits plasticity in adult visual cortex. Science 330: 1238-1240.

Murthy VN, Schikorski T, Stevens CF, Zhu Y. 2001. Inactivity produces increases in neurotransmitter release and synapse size. Neuron 32: 673-682.

Philpot BD, Sekhar AK, Shouval HZ, Bear MF. 2001. Visual experience and deprivation bidirectionally modify the composition and function of NMDA receptors in visual cortex. Neuron 29: 157-169.

Pitsch J, Opitz T, Borm V, Woitecki A, Staniek M, Beck H, Becker AJ, Schoch S. 2012. The presynaptic active zone protein RIM1 $\alpha$ controls epileptogenesis following status epilepticus. J Neurosci 32: 12384-12395.
Pizzorusso T, Medini P, Berardi N, Chierzi S, Fawcett JW, Maffei L. 2002. Reactivation of ocular dominance plasticity in the adult visual cortex. Science 298: $1248-1251$.

Quinlan EM, Philpot BD, Huganir RL, Bear MF. 1999. Rapid, experience-dependent expression of synaptic NMDA receptors in visual cortex in vivo. Nat Neurosci 2: 352-357.

Reyes A, Lujan R, Rozov A, Burnashev N, Somogyi P, Sakmann B. 1998. Target-cell-specific facilitation and depression in neocortical circuits. Nat Neurosci 1: 279-285.

Rodríguez-Moreno A, Paulsen O. 2008. Spike timing-dependent long-term depression requires presynaptic NMDA receptors. Nat Neurosci 11: $744-745$

Rodríguez-Moreno A, Kohl MM, Reeve JE, Eaton TR, Collins HA, Anderson HL, Paulsen O. 2011. Presynaptic induction and expression of timing-dependent long-term depression demonstrated by compartment-specific photorelease of a use-dependent NMDA receptor antagonist. J Neurosci 31: 8564-8569.

Rodríguez-Moreno A, González-Rueda A, Banerjee A, Upton AL, Craig MT, Paulsen O. 2013. Presynaptic self-depression at developing neocortical synapses. Neuron 77: $35-42$.

Rozov A, Burnashev N, Sakmann B, Neher E. 2001. Transmitter release modulation by intracellular $\mathrm{Ca}^{2+}$ buffers in facilitating and depressing nerve terminals of pyramidal cells in layer $2 / 3$ of the rat neocortex indicates a target cell-specific difference in presynaptic calcium dynamics. J Physiol 531: 807-826.

Sah P, Hestrin S, Nicoll RA. 1989. Tonic activation of NMDA receptors by ambient glutamate enhances excitability of neurons. Science 246: 815-818.

Salter MG, Fern R. 2005. NMDA receptors are expressed in developing oligodendrocyte processes and mediate injury. Nature 438: 1167-1171.

Scimemi A, Schorge S, Kullmann DM, Walker MC. 2006. Epileptogenesis is associated with enhanced glutamatergic transmission in the perforant path. J Neurophysiol 95: 1213-1220.

Shu Y, Hasenstaub A, Duque A, Yu Y, McCormick DA. 2006. Modulation of intracortical synaptic potentials by presynaptic somatic membrane potential. Nature 441: $761-765$

Sjöström PJ, Turrigiano GG, Nelson SB. 2003. Neocortical LTD via coincident activation of presynaptic NMDA and cannabinoid receptors. Neuron 39: 641-654.

Tovar KR, Westbrook GL. 1999. The incorporation of NMDA receptors with a distinct subunit composition at nascent hippocampal synapses in vitro. J Neurosci 19: 4180-4188.

Turrigiano GG, Leslie KR, Desai NS, Rutherford LC, Nelson SB. 1998. Activity-dependent scaling of quantal amplitude in neocortical neurons. Nature 391: 892-896.

Upreti C, Otero R, Partida C, Skinner F, Thakker R, Pacheco LF, Zhou ZY, Maglakelidze G, Velíšková J, Velíšek L, et al. 2012. Altered neurotransmitter release, vesicle recycling and presynaptic structure in the pilocarpine model of temporal lobe epilepsy. Brain 135: 869-885.

Wen JA, Barth AL. 2011. Input-specific critical periods for experience-dependent plasticity in layer $2 / 3$ pyramidal neurons. J Neurosci 31: 4456-4465.

Wen JA, Barth AL. 2012. Synaptic lability after experience-dependent plasticity is not mediated by calcium-permeable AMPARs. Front Mol Neurosci 5: 15.

Wen JA, DeBlois MC, Barth AL. 2013. Initiation, labile, and stabilization phases of experience-dependent plasticity at neocortical synapses. J Neurosci 33: 8483-8493.

Yang J, Woodhall GL, Jones RS. 2006. Tonic facilitation of glutamate release by presynaptic NR2B-containing NMDA receptors is increased in the entorhinal cortex of chronically epileptic rats. J Neurosci 26: 406-410.

Yashiro K, Philpot BD. 2008. Regulation of NMDA receptor subunit expression and its implications for LTD, LTP, and metaplasticity. Neuropharmacology 55: 1081-1094.

Yoshimura Y, Ohmura T, Komatsu Y. 2003. Two forms of synaptic plasticity with distinct dependence on age, experience, and NMDA receptor subtype in rat visual cortex. J Neurosci 23: 6557-6566.

Zhou Y, Li HL, Zhao R, Yang LT, Dong Y, Yue X, Ma YY, Wang Z, Chen J, Cui CL, et al. 2010. Astrocytes express $N$-methyl-D-aspartate receptor subunits in development, ischemia and post-ischemia. Neurochem Res 35: $2124-2134$.

Received May 16, 2014; accepted in revised form September 25, 2014. 


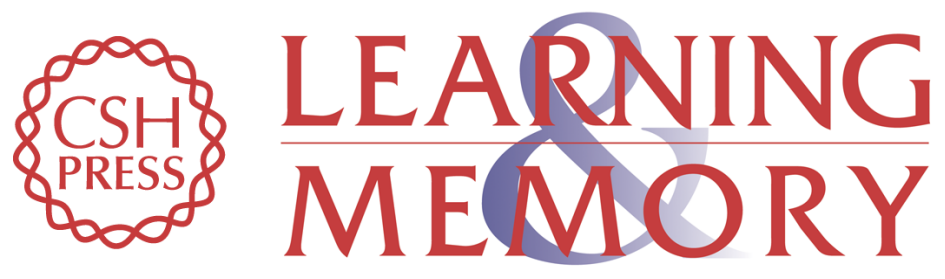

\section{Experience-dependent regulation of presynaptic NMDARs enhances neurotransmitter release at neocortical synapses}

Joanna Urban-Ciecko, Jing A. Wen, Puja K. Parekh, et al.

Learn. Mem. 2015, 22:

Access the most recent version at doi:10.1101/lm.035741.114

Supplemental http://learnmem.cshlp.org/content/suppl/2014/12/05/22.1.47.DC1
Material

References This article cites 72 articles, 28 of which can be accessed free at: http://learnmem.cshlp.org/content/22/1/47.full.html\#ref-list-1

Creative This article, published in Learning \& Memory, is available under a Creative Commons Commons License (Attribution-NonCommercial 4.0 International), as described at License http://creativecommons.org/licenses/by-nc/4.0/.

Email Alerting Receive free email alerts when new articles cite this article - sign up in the box at the Service top right corner of the article or click here. 